\title{
The Effects of Font Size and Page Presentation Method of E-Book Reading on Small Screens for Older Adults
}

\author{
Wang-Chin Tsai, Yi-Lin Ro, Ya-Tzu Chang, and Chang-Franw Lee \\ Graduate School of Design, National Yunlin University of Science and Technology \\ 123, University Road Section 3, Touliu, Yunlin, 64002, Taiwan, R.O.C. \\ g9330802@yuntech. edu.tw
}

\begin{abstract}
It is predictable that the E-book market would attract more and more older customers, and has the potential benefits for older adults to interact with technology and enjoy the reading pleasure. The main purpose of this research is to investigate the effects with font sizes and page presentation methods for the E-Book reading performance. 24 older adults, ages 62 to 70, participated in the study. Reading speed and accuracy were measured and subjective satisfaction assessment of older adults recorded. The results indicate that reading with flip page method of E-book caused significantly higher reading speed than reading with scrollbar page method. Subjective results showed that older adults preferred 14 font size and more positive comments showed a preference with flip page presentation method.
\end{abstract}

Keywords: Older adults, E-book, Font size, Page presentation method.

\section{Introduction}

Recently, the "E-book" was introduced into the market as one kind of personal digital information service. Total sales in 2003 amounted to $\$ 10$ million representing an increase of more than 32\% over 2002 [1]. The E-book is a new information technology product that facilitates reading and acquisition of information. It is a written work readable on the screen of PC or handheld device: mobile phone, PDA (personal digital assistant), and reader specifically designed for the purpose (I-Pad, Amazon Kindle, Nook). Different company also developed various applications for the E-book reading platform (iBook, B\&N eReader, Kindle, Stanza, Kobo, etc). Under this trend, more and more people would like to start changing their reading habit to read the E-book via different kinds of device. Also, usability research studies about E-book issue focused on investigating the effect of reading performance from paper and screen and the relationship between reading speed, accuracy, comprehension, fatigue, preference and reading performance. Mayes et al. [2] showed that subjects take longer to read text on a screen than on paper. As for accuracy, Egan et al. [3] reported that students using digital hypertext on a screen to find out specific information in the text had higher accuracy than students using the paper text. For reading comprehension, Dillon and Gabbard [4] concluded that comprehension when reading from a screen is better than reading from paper when performing substantial searching or manipulation and 
comparison of visual details among objects. Some studies have investigated on how to display textual information on small screens. For example, presenting text dynamically on screens (e.g. vertical scrolling) [5] and analyzing web design guidelines for applicability to small screen interfaces [6]. Recommendations for text sizes from previous studies have indicated font size 14 for children [7], font size 14 for older adults [8], and font size 12 for young to middle-aged adults [9]. The Kang compared the differences between reading an E-book and a conventional book (C-book) with objective measures. The results indicate that reading an E-book causes significantly higher eye fatigue than reading a C-book [10]. Furthermore, Siegenthaler et al. [11] investigated the requirements of e-reader design and tested five electronic reading devices and one classic paper book. The results showed that the current e-reader generation has large deficits with respect to usability. Users were unable to use e-readers intuitively and without problems.

Therefore, developers followed these research results and better designed a series of E-book reading application to fit for personal reading requirements and improve the insufficient parts for the E-book interface. The main goal is to provide a reading experience as close as possible to the clarity and the lack of glare found with print books. Current E-book interface offered some tailored functions: Font, sizes, text background, page flip type, and page layout for users to adjust. Ideally, such an Ebook reading would allow the user to pick the most comfortable presentation and be able to reflow the content for that setting. Furthermore, a lightweight E-book reading with friendly interface would be easy for users to hold and manipulate.

However, apart from the current usability studies and implications about E-book, seldom researches and developers had focused on the older adults readers. As the oldest Baby Boomers become senior citizens in 2011, the population 65 and older is projected to grow faster in Taiwan. According to the investigation of older adult' leisure activities in Taiwan, reading newspaper and book is one of the major recreation in their daily life [12]. It is predictable that the E-book market would attract more and more older customers, and has the potential benefits for older adults to interact with technology and enjoy the reading pleasure. Furthermore, these portable and handheld e-book approaches are also convenient for older adults to catch the information at their preferred time and place. However, in Taiwan, most of the current E-book readers are with small screen, like mobile phone and PDA. A lot of aging decline changes on motor, perceptual and cognitive abilities could affect the text reading and performance on the small screen reader device. Older adults may struggle with Ebooks, magazines and newspapers because of the declined aging effects.

As mentioned before, the previous studies were targeted on younger adults with computer or other small screen device usage, but few data are available on how different variables vary with E-book reading performance and comprehension for older adults. Under this circumstance, we need to have an insight on the E-book interface that is easy to use and quick to learn for older adults. Apparently, the E-books might be the solution in many cases with appropriate design and resolutions available for older adults as well. Many older adults will not consider an E-book interface if they have to invest several hours or days to learn how to use it.

In this paper, we chose the mobile phone as an E-book reader device. The reason is the current released E-book approaches are popular in this device, and it corresponds for the small screen device features. Reading E-book from an electronic interface of 
small screen is completely different than reading a conventional book. The reading behavior and visual demand when using an E-book require further study. In this study, we selected two most common elements (font size and page presentation method) as the variables on the base of previous E-book research studies. We aim to elicit an indication of a suitable font size to use under different page presentation methods on small screen devices and determine whether concrete font sizes and page presentation methods are required when designing E-book for older people. Therefore, it may be expected that there will be differences of font size, page presentation methods and reading performance on small screen display, especially for older adults. We also investigate the user satisfaction to get better understanding for the subjective evaluation on E-book reading under different situations. The next section outlines the experiment used in this study. The results from the experiment are then presented and discussed. Some areas for further investigation are suggested. Finally the conclusions drawn from our experiences are given.

\section{Methods}

The experiment was a $3 \times 2$ factor between-subjects design. The first factor was font size (three levels: 10, 12, and14 point) and the second factor was page presentation method (two levels: Scrollbar page and Flip page, Figure.1). Based on the factorial design, there are totally six articles composed of designated levels for the experiment. One article assigned four participants to read. Their response measures included reading performance (speed and accuracy) and subjective satisfaction assessment.

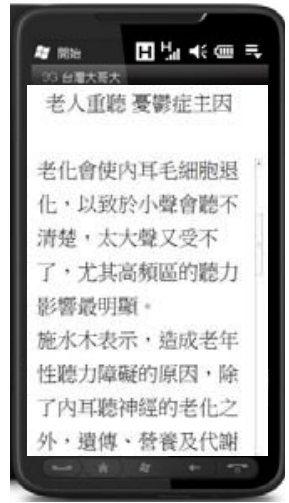

(a)

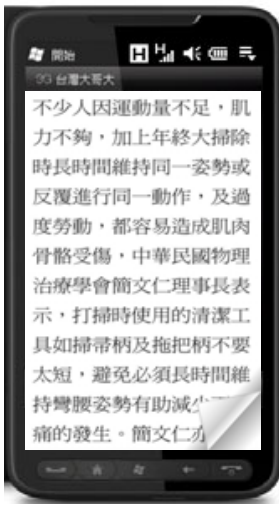

(b)

Fig. 1. The illustration of page presentation method: (a) scrollbar page and (b) flip page used in this study

\subsection{Participants}

In this study, we invited 24 older adults took part in the E-book reading performance test and satisfaction investigation. All participants were fluent in Chinese as their first 
language and educated to at least secondary/high school level. The 12 females and 12 males were between the ages of 60 and 75 years (mean $=64.8, \mathrm{SD}=5.72$ ). They were required to have at least $20 / 25$ visual acuity with corrective lenses and to be without physical or mental problems. They were also requested not to stay up late, take medicine, alcoholic drinks and any other substance that might possibly affect the test results. All subjects had no previous experience using the E-book. A small gift was given to participants as payment for taking part.

\subsection{Equipment}

An HTC HD2 (www.htc.com.tw) which has a 65,000 color TFT capacitive touch screen with a resolution of $480 \times 800$ pixels was used to present the E-book text (see Figure 1). This has the optimal quality screen available. The screen was backlit and participants sat in a usability lab which was illuminated by overhead fluorescent lights. The HTC HD2 used the Microsoft Windows Mobile 6.5 professional operating system and had Clear Type enabled to anti-alias the edges of fonts to improve quality. The touch screen could allow the participants use the finger to control the paper turning function. Adobe Flash software was used to present the experimental texts and page presentation method. An example of the software running on the HTC HD2 is shown in Figure 1.

\subsection{Materials}

The standard Microsoft Ming type Chinese characters was chosen for displaying text since it has been found that is the most frequently used font for Chinese textual information and is also known as the standard writing type. Text was presented to participants at font sizes 10pt、12pt and 14pt. Examples of each size are shown in Figure 1.

Two different page presentation methods of E-book were used. The "scrollbar page" is a page function used frequently in a graphical user interface (GUI) with which continuous text, pictures or anything else can be scrolled. Scrollbars are present in a wide range of electronic devices including computers, graphing calculators, mobile phones, and portable media players. They usually appear on one or two sides of the viewing area as long rectangular areas containing a bar (or thumb) that can be dragged along a trough (or track) to move the body of the document as well as two arrows on either end for precise adjustments. In the E-book reading, participant can use their finger to drag the bar for turning pages back and forth.

Flip page refers to the effect of flipping through the pages of a digital document as if it were a physical document. A flip page application is often made in Adobe Flash and requires the Adobe Flash Player to run in a browser interface. The benefit of having a flip page document is that it affords the user experience of reading an actual copy of a physical document or magazine. The technology is commonly used by traditional publishers that want to create (and spread) a digital version of their physical document/paper/magazine.

\subsection{Reading Performance and Subjective Satisfaction}

In the experiment, all subjects read one articles as the E-book demonstration test. Each subject spent about 5 to 10 minutes to read passages page by page with an average of 
Chinese characters in each article. There were three reading test questions after article reading. The test was recall-type questions. For example, "What are the critical ingredients could promote assimilated calcium for human beings?" or "What are the best prepared time for preventing suffered from Osteoporosis?" Subjects were required to answer correct selections for specific information in the text. Both reading speed and reading accuracy (proportion of correct recall) were taken as performance measures.

\subsection{Experiment Procedure}

A standard laboratory desk and chair were provided for experimentation. The experiment environment was standardized. Prior to the experiment each participant was instructed about the purpose and procedure of the study. Participants were asked hold the HD2 and to read the passages from a comfortable position and were told that they could bring the HD2 closer to the face if necessary. The article was randomized for each participant. At the beginning of each reading session, the participant was then asked to read one health-care issues article and then take a reading recall test. Instructions were given to only say the erroneous word(s) and nothing else while reading a passage and keep questions/comments for the breaks between passages. Both reading speed and reading accuracy (proportion of correct recall) were measured. After being presented with an initial set of 6 articles to read, participants were requested to answer the subjective satisfaction on what they thought of the different text sizes and page presentation method of the E-book by 5 point Likert scale. For the analysis of the data, this study applied Two-way Analyses of Variance (ANOVA) to examine significant differences of the reading performance and accuracy. In addition, the significant differences were analyzed by utilizing the Scheffe Method as the post hoc test for multiple comparisons. Significance was accepted at the level of $\mathrm{p}<.05$, while the degrees of freedom and corresponding probability, or the F-value, were also shown in the statistical test. In all, the statistical analysis was conducted by utilizing the Windows SPSS Statistics 18 Program.

\section{Results}

\subsection{Reading Performance}

Reading performance was measured by reading speed and reading accuracy (proportion of correct recall). Table 1 shows the average performance of reading on E-books. These findings indicated that there is no significant interaction effect among the reading speed $[F(2,18)=0.93, p>.05]$ and reading accuracy $[F(2,18)=0.60, p>.05$, in all cases] for older adults in the experiment. The effects of two independent variables, Font size and page presentation method, were explored (Table 2). The analysis of variance indicated that only the page presentation method $[\mathrm{F}(1,18)=5.60, \mathrm{p}<.05]$ affected reading speed of the older adults. Though there was a slight decrease in reading time at font size 14 it was not statistically significant. Therefore, we continue perform the post hoc multiple comparisons in order to understand the difference between these two page presentation method level. The post hoc multiple comparisons were integrated and are shown in tables 3 in this study. 
Table 1. The ANOVA results

\begin{tabular}{lll}
\hline \multirow{2}{*}{ Factor } & \multicolumn{2}{c}{ Reading Performance } \\
\cline { 2 - 3 } & Reading Speed & Reading accuracy \\
\hline Font Size (A) & - & - \\
Page Presentation Method (B) & $*$ & - \\
\hline$: \mathrm{p}<0.05$. & &
\end{tabular}

Table 2. Significant analysis of two independent variables on reading speed

\begin{tabular}{llllll}
\hline Source of Variances & Sum of square & Df & MS & F & P value \\
\hline Text Size (A) & 4823.5 & 2 & 2411.7 & 0.74 & 0.487 \\
Page Presentation Method (B) & 16287.5 & 1 & 16287.5 & 5.60 & $0.037^{*}$ \\
A * B & 5994.05 & 2 & 2997.02 & 0.93 & 0.412 \\
Error $(A *$ B ) & 57932.07 & 18 & 3218.4 & & \\
\hline
\end{tabular}
$*: \mathrm{p}<0.05$.

Table 3. Results of reading speed on page presentation method and text size

\begin{tabular}{clllll}
\hline \multirow{2}{*}{ Source of Variances } & \multicolumn{2}{l}{ Presentation Method* } & \multicolumn{3}{c}{ Font Sizes } \\
\cline { 2 - 6 } & Scrollbar & Flip Page & 10 point & 12 point & 14 point \\
\hline Time(unit: seconds) & 134.86 & 82.75 & 115.77 & 121.61 & 89.04 \\
\hline
\end{tabular}

According to the results of the ANOVA, there was a significant difference with the page presentation method on the reading speed of total times. For both page presentation methods, the performance of total time was larger on the scrollbar page than on flip page method (Table 3 ).

\subsection{Subjective Satisfaction Results}

It is important to consider whether there are no any significant differences between font size and old participant's subjective satisfaction or whether preferences change between scrollbar page and flip page. However, older adults' comments on specific font size and flip page presentation method were examined and recorded on protocol content. Older adults expressed that they preferred 14 point font size with flip page presentation method, received more desirable comments, both fairly neutral.

Furthermore, the majority of $70 \%$ of participants also agreed that flip page presentation method was desirable for the E-book usage. On the other hand, scrollbar page had a negative rated from older adults, especially while font size 10 was considered.

\section{Discussions}

The results show that objective measures (time and accuracy) of reading performance are not affected significantly by changes in font size, but only affected by the page presentation method for older adults. Even the time needed to read the text and the reading accuracy did not differ between the different font sizes. It could also be men- 
tioned the fact that participants had the possibility to get accustomed to the current font size probably on a small screen display. However, older adults still complained about reading the small text on the E-book is tired and exhausted. It could because of reading distance was inconstant and font size in the E-book was very small, some participants (especially older participants) reported problems with reading. The possible reason for the time increased could due to the eye fatigue with small font size. Therefore, larger font size can efficiently lower eye-movement fatigue and lead to better usability and also to better legibility in the E-book reading. Otherwise, as font size increases the number of pages will add. Increasing font size inevitably results in a higher number of pages and, as a consequence, more page-turns. Interestingly, older adults do not afraid turning too many pages, but did feel frustrated with the small font sixe. Even though there was no significant effect on reading performance, the big font size of the E-book was somewhat preferred than that of the small font size.

Actually, in our research, the reading performance was only affected by the page presentation method. Flip page presentation method take a shorter time than the scrollbar page method. Obviously, the digital displays of E-books have become more sophisticated and are increasingly realistic present. The visual appearance with flip page is intuitive for the older adults. Therefore, older adults can turn page easily between pages. In particular, older adults responded the page-turns with scrollbar still take more delayed seconds. The scrollbar page were required the older adults to achieve higher manual dexterity demand. Because of the aging effects on hand ability, it is hard to scroll a "page" slightly while finish the reading page. A possible explanation is that even the scrollbar function is a common function in electronic interface, it could be a different situation used on a small touch screen E-books, which requires more detailed scrolling action within the passages changes.

The E-book technology for small screen devices is changing rapidly for the better. The e-link technique used in the future version of the readers make the text and characters much easier to see at small sizes. This indicates that our findings may not be applicable to future displays with improved resolutions. However, our findings that a bigger font sizes is preferred by older adults are likely to be the same for future screens, with the bounds of the range changing with resolution changes.

\section{Conclusions}

This study evaluated the performance and subjective satisfaction of reading an E-book with two kinds of variables (font size and page presentation method). Although there were no significant differences on font size in reading performance or accuracy due to aging deficiency, there was variation in older adults' subjective preferences on the font size used. The results indicate that page presentation method caused significantly difference on higher performance with than scrollbar page in E-reading. This is mainly due to the intuitive concept of the display for an E-book. Since the reading habit for E-books was different with conventional books, especially for older adults. There are more issues needed to take into consideration. Moreover, E-book reading will become a promising trend for future development. Therefore, the establishment of current research results for optimized E-book design is of great significance. 


\section{References}

1. Boss, R.W.: E-book; An uncertain future, Tech Notes, American Library Association (2004)

2. Mayes, D.K., Sims, V.K., Koonce, J.M.: Comprehension and workload differences for VDT and paper-based reading. International Journal of Industrial Ergonomics 28(6), 367378 (2001)

3. Egan, D.E., Remde, J.R., Landauer, T.K., Lochbaum, C.C., Gomez, L.M.: Behavioral evaluation and analysis of a hypertext browser. In: Proceedings of CHI 1989, pp. 205-210. Association for Computing Machinery, New York (1989)

4. Dillon, A., Gabbard, R.: Hypermedia as an educational technology: a review of the quantitative research literature learner comprehension, control, and style. Review of Educational Research 68(3), 322-349 (1998)

5. Laarni, K.: Searching for Optimal Methods of Presenting Dynamic Text on Different Types of Screens. In: Proceedings of the Second Nordic Conference on Human-Computer Interaction, pp. 219-222. ACM Press, New York (2002)

6. Karkkainen, L., Laarni, J.: Designing for Small Display Screens. In: Proceedings of the Second Nordic Conference on Human-Computer Interaction, pp. 227-230. ACM Press, New York (2002)

7. Bernard, M.L., Chaparro, B.S., Mills, M.M., Halcomb, C.G.: Examining Children's Reading Performance and Preference for Different Computer-Displayed Text. Journal of Behaviour and Information Technology 21(2), 87-96 (2002)

8. Bernard, M.L., Liao, C.H., Mills, M.M.: The Effects of Font Type and Size on the Legibility and Reading Time of Online Text by Older Adults. In: Proceedings of ACM CHI 2001, vol. II, pp. 175-176. ACM Press, New York (2001)

9. Bernard, M.L., Chaparro, B.S., Mills, M.M., Halcomb, C.G.: Comparing the Effects of Text Size and Format on the Readability of Computer-Displayed Times New Roman and Arial Text. International Journal of Human-Computer Studies 59, 823-835 (2003)

10. Kang, Y., Wang, M.J., Lin, R.: Usability evaluation of E-books. Displays 30(2), 49-52 (2009)

11. Siegenthaler, E., Wurtz, P., Groner, R.: Improving the Usability of E-Book Readers. Journal of Usability Studies 6(1), 25-38 (2010)

12. Ministry of Interior, Report of the Senior Citizen Condition Survey 2009, Republic of China (2009) 